set of positive integers $\delta_{i}$ such that $Q_{1}\left(y_{0}, y_{1} \delta_{1}, \cdots, y_{v} \delta_{\nu}\right)$ would be resolvable into more than $K_{m}$ factors, which is not the case. Each of the functions (7) is a factor of $f(x)$.

When we multiply together the simple functions coming from the irreducible binomial factors of $Q$ which do not involve $y_{0}$ and the irreducible functions coming from the remaining irreducible factors of $Q$, we have a resolution of $f(x)$ into factors belonging to the class $C$. It is easily seen that this factorization is unique. Thus we have the following theorem.

Theorem. A function $f(x)$ belonging to the class $C$ can be expressed in one and only one way as a product

$$
f(x)=I_{1}(x) \cdots I_{m}(x) S_{1}(x) \cdots S_{n}(x),
$$

where each factor belongs to $C$, the I's are irreducible functions, and the $S$ 's are simple functions, $b_{0}+\sum b_{i} \exp \left(\beta_{i} x\right)$, such that the ratio of any two $\beta$ 's in different functions is irrational.

Bell Telephone Laboratories

\title{
THE NUMBER OF TRISECANTS OF A SPACE CURVE OF ORDER $m$ WHICH MEET AN $i$-FOLD SECANT*
}

BY L. A. DYE

The number of trisecants of a space curve $C_{m}$, of order $m$, which meet a general line was determined by Zeuthen, $\dagger$ but if the line happens to be an $i$-fold secant, $i>2$, it lies on the ruled surface of trisecants and the formula fails. In algebraic geometry some extension of Zeuthen's work to cover this neglected case is often necessary, so by means of a correspondence we show that the number of trisecants of a $C_{m}$ which meet an $i$-fold secant $l$ is

$(m-2)[h-m(m-1) / 6]-i(h-m+2)+i(i-1)(i-2) / 6$, where $h$ is the number of apparent double points of $C_{m}$.

In the plane determined by $l$ and one of the $h^{\prime}=h-i(i-1) / 2$

* Presented to the Society, October 27, 1934.

$\dagger$ H. G. Zeuthen, Sur les singularités des courbes gauches, Annali di Matematica, (2), vol. 3 (1869), pp. 175-217. 
bisecants of $C_{m}$ through any point $\lambda$ on $l$, let $A, B$ be the points of $C_{m}$ on the bisecant and let $C_{j},[j=1,2, \cdots,(m-i-2)]$, denote the points of $C_{m}$ not on $l$ or the bisecant. The lines $A C_{j}$ and $B C_{j}$ determine $2(m-i-2)$ points $\mu$ on $l$, and when all of the $h^{\prime}$ bisecants through the point $\lambda$ are considered there are $2(m-i-2) h^{\prime}$ points $\mu$ determined by each point $\lambda$. Since the relationship between the points $\lambda$ and $\mu$ is symmetrical, there exists a $\left[2(m-i-2) h^{\prime}, 2(m-i-2) h^{\prime}\right]$ correspondence. The $4(m-i-2) h^{\prime}$ coincidences of this correspondence fall into three classes.

1. In the plane determined by $l$ and a trisecant of $C_{m}$ meeting $l$ let $A, B, C$ be the points of $C_{m}$ on the trisecant. If the line is thought of as $A B$, then $A C$ and $B C$ each account for a coincidence. Similarly the line may be taken as $A C$ or $B C$, so that 6 coincidences arise from each of the $x$ trisecants of $C_{m}$ meeting $l$.

2. Since there are $r=m(m-1)-2 h$ tangents to $C_{m}$ meeting an arbitrary line, there are $r^{\prime}=r-2 i$ tangents meeting $l$. In the plane of one of these tangents and $l$, let $A \equiv B$ be the point of tangency and $C_{j}$ any one of the $m-i-2$ residual intersections of $C_{m}$ not on $l$. For each line $A C_{j}$ there arises one coincidence due to $B C_{j}$, hence there are $(m-i-2) r^{\prime}$ coincidences due to the tangent lines meeting $l$.

3. In the plane determined by $l$ and a tangent to $C_{m}$ at one of the $i$ intersections with $l$, call the point of tangency $A \equiv B$. Join $A$ to one of the $m-i-1$ residual intersections $C_{j}$ of $C_{m}$, then the lines joining $B$ to the $m-i-2$ remaining points $C_{k}$ determine $m-i-2$ coincidences. Since there are $m-i-1$ choices for $C_{j}$, and $i$ points on $l$, there are $i(m-i-1)(m-i-2)$ coincidences accounted for in this case.

We now solve the equation $4(m-i-2) h^{\prime}=6 x+(m-i-2) r^{\prime}+i(m-i-1)(m-i-2)$, and obtain

$$
x=(m-2)[h-m(m-1) / 6]-i(h-m+2)+i(i-1)(i-2) / 6 .
$$

Cornell University 